\title{
Formulation and performance of variational integrators for rotating bodies
}

\author{
Ignacio Romero
}

\begin{abstract}
Variational integrators are obtained for two mechanical systems whose configuration spaces are, respectively, the rotation group and the unit sphere. In the first case, an integration algorithm is presented for Euler's equations of the free rigid body, following the ideas of Marsden et al. (Nonlinearity 12:1647-1662, 1999). In the second example, a variational time integrator is formulated for the rigid dumbbell. Both methods are formulated directly on their nonlinear configuration spaces, without using Lagrange multipliers. They are one-step, second order methods which show exact conservation of a discrete angular momentum which is identified in each case. Numerical examples illustrate their properties and compare them with existing integrators of the literature.
\end{abstract}

Keywords Time integration • Rigid body • Rotations • Variational method $\cdot$ Geometric integration

\section{Introduction}

Certain important mechanical systems are formulated on nonlinear configuration spaces and this non-linearity greatly complicates their numerical approximation. For example, in structural mechanics, geometrically exact rods and shells have configuration spaces that include the space of rotations and the unit sphere, respectively The rigid body and the dumbbell furnish simpler models whose configuration spaces are also the rotation group and the unit sphere, respectively, and their analysis provides insight into the behavior of the more complicated structural models mentioned above. These two model problems are, moreover, important in their own right, since they appear frequently in many Engineering applications, such as robotics, multibody systems, vehicle dynamics, etc.

When solving numerically any of these problems it is important to identify and select the most accurate and robust time integration schemes available, and many articles have been devoted to the development of such type of methods

The concept of "accuracy" needs to be properly understood. Whereas the order of accuracy of a given algorithm is an unambiguous concept, there are other important aspects that come into play when trying to ascertain the "correctness" and precision of a given numerical solution. Some of these are the preservation of invariants (such as energy and momentum) and other qualitative features of the exact flow (relative equilibria, symplecticity, etc). In long term dynamic simulations, numerical errors unavoidably accumulate and can add up to a significant part of the solution. For this type of problems, even algorithms that possess high order of accuracy can provide essentially wrong responses. In these cases it is especially important to use algorithms which capture accurately the qualitative features of the solution. It might be advisable to select an algorithm with low order of accuracy versus another with higher order if the former produces a more "correct" and thus more "accurate" picture of the solution dynamics.

Arguments of this sort have motivated the current interest in so called geometric integrators that is, methods that capture some of the qualitative properties of the exact motion. Some of the most popular geometric integrators for Hamiltonian systems such as the rigid body include symplectic methods and the energy-momentum methods 
Variational integrators, a family of geometric methods whose origins can be traced back to, are becoming increasingly popular and an active area of current research

The idea underlying these methods is strikingly simple: instead of discretizing the ordinary differential equations of motion, variational integrators seek the stationary solution of a discrete action functional. In order to do so, only an approximation of the Lagrangian is required which, moreover, must be expressed solely in terms of the discrete positions of the system. Once the Lagrangian is chosen, the dynamic equations follow systematically. These equations have, by construction, symmetries and conservation laws related to those of the continuum equations.

The equations of a variational integrator depend exclusively on the choice of the discrete Lagrangian. Using it, a set of discrete Euler-Lagrange equations can be derived which provide algorithmic updates that generate a discrete flow. In mechanical systems with linear configuration spaces, for example, nonlinear systems of springs and point masses, the formulation of the discrete Lagrangian is trivial, and their corresponding Euler-Lagrange equations are readily obtained

In contrast, formulating a discrete Lagrangian for a mechanical system with a nonlinear configuration space is not so simple

Despite these difficulties, and given the success of variational methods and their potential, it seems relevant to apply them in these nonlinear spaces. The original work of already presented a variational method for the rigid body equations. This idea was further exploited in It was found in the latter work that such an algorithm could be implemented using quaternion variables and a Lagrange multiplier to enforce the constraint that the quaternions should remain of unit modulus.

In this work we will present variational integrators for the Euler equations of rigid body dynamics and the dumbbell formulated directly on their nonlinear configuration manifolds. In the case of the rigid body, some of these ideas have been presented in abstract form in without details on the implementation nor numerical results. In contrast, in this article we will provide explicit expressions of the discrete update maps that define variational integrators in two nonlinear spaces, and we will assess their performance in terms of "accuracy", as discussed above, comparing them with other existing methods.

An outline of the rest of the paper is as follows. Section 2 reviews some basic concepts related to the mathematical description of the rotation group and its relation with the unit sphere. In Sect. 3, Euler's equations of the rigid body are obtained from the Lagrangian formalism, illustrating the continuous variational framework of the equations. Next, in Sect. 4 the variational integrator for the former equations is presented, and its properties are discussed. Sections 5 and 6 discuss the continuous and discrete variational formulation of the rigid dumbbell, mimicking the analysis devoted to the rigid body. The numerical properties of all the proposed methods are verified and illustrated by means of numerical examples in Sect. 7. A summary of the main results is provided in Sect. 8.

\section{The rotation group and the unit sphere}

Euler's equations of motion are formulated in the special orthogonal group of rotation matrices, the Lie group $\mathrm{SO}(3)$. Some notation and a few definitions regarding this group will be useful for the derivation of the algorithms of later sections.

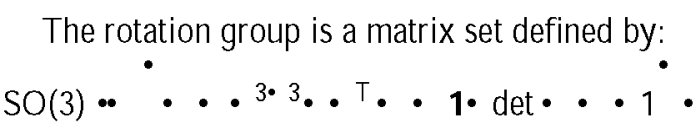

together with the operation of matrix multiplication. The Lie algebra of $\mathrm{SO}(3)$ is denoted so(3) and corresponds to the space of infinitesimal rotations, represented by the skewsymmetric matrices:

so(3) • ' $\mathbf{w} \cdot 3 \cdot 3 \cdot \mathbf{w}^{\top} \cdot \mathrm{w}^{\bullet} \cdot$

There is an isomorphism between skew-symmetric matrices and vectors. Each skew matrix $\mathbf{W}$ has a unique axial vector $\mathbf{W}$ such that $\mathbf{W} \mathbf{b} \cdot \mathbf{W} \cdot \mathbf{b}$, for all $\mathbf{b} \cdot \boldsymbol{B}^{3}$.

The exponential map exp [•] takes skew matrices into rotation matrices and its close form is given by Rodrigues' formula:

$$
\begin{aligned}
& \exp \ddot{\mathbf{a}} \cdot \mathbf{1} \cdot \frac{\sin \cdot \mathbf{a} \bullet}{\mathbf{a}^{\bullet}} \mathbf{a} \cdot \frac{1}{2} \cdot \frac{\sin _{\frac{a_{\bullet}}{2}}^{\bullet 2}}{\frac{\mathbf{a}^{\bullet}}{2}} \cdot \mathbf{a}^{2} \\
& \because \bullet^{1} \cdot \exp \cdot \mathbf{a} \text { can be easily verified }
\end{aligned}
$$

The property $\exp \because{ }^{1} \cdot \exp \cdot \mathbf{a}$ can be easily verified.
Frequently, it will be necessary to refer to incremental rotations and rotation vectors. Given two rotations $\boldsymbol{~}_{1} \boldsymbol{\bullet}_{2}$ we define the (right) incremental rotation $\mathbf{R}_{2}^{1}$ and its associated (right) incremental rotation vector $-{ }_{2}^{1}$ as

$$
\mathbf{R}_{2}^{1} \cdot \bullet_{1}^{\top} \cdot{ }_{2} \cdot \bullet_{2}^{1} \cdot \exp ^{\bullet}{ }^{1} \cdot \mathbf{R}_{2}^{1} \cdot \bullet
$$

In the derivation of the equations of the integrator it will be required to take derivatives and variations of curves in $\mathrm{SO}(3)$. For that, given a curve in $\mathrm{SO}(3)$ parametrized by time $\cdots+\cdots 0 \cdot T \cdot S \mathrm{SO}(3)$, the time derivative of $\cdots \cdot \bullet$ can be written as:

$$
\bullet \cdot \frac{\mathrm{d}}{\mathrm{dt}} \cdot \bullet \mathrm{t} \cdot \cdots \cdot \bullet \cdot \bullet \cdot \bullet \cdot \bullet
$$

where $\cdot \bullet t \cdot$ is a skew-symmetric matrix. Similarly, any variation of this curve must be of the form

$\cdots \cdot \cdot \cdot \cdot \cdot \cdots \cdot \cdot \cdot \cdot \cdot$

for some arbitrary curve $\cdot \bullet^{\bullet} \cdot \bullet \cdot$ in $\mathrm{SO}(3)$. 
Finally, we will need to linearize the exponential map If $\bullet \cdot \bullet$ is a function is $\mathrm{SO}(3)$ of real variable $t$ then

$$
\frac{\mathrm{d}}{\mathrm{dt}} \exp \bullet \bullet \cdot \bullet \cdot \operatorname{cexp} \bullet \cdot \cdot \cdots \bullet \cdot \bullet \cdot \exp \bullet \bullet \cdot \bullet
$$

where the operator dexp $\cdots$ is defined by

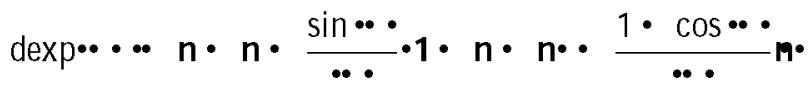

with $\mathbf{n} \cdot \stackrel{\bullet}{\cdots \cdot} \cdot$

In this last expression, and for the rest of the section, the dependence of - on t is not explicitly shown. The inverse of the operator dexp... has a close form expression that reads:

dexpinv $\cdots \cdot \cdots \operatorname{dexp} \cdot \bullet^{\bullet} 1$

$$
\text { - } n \cdot n \cdot \frac{\frac{\ddot{\theta}}{2}}{\tan \frac{\ddot{\theta}}{2}} \cdot \mathbf{n} \cdot \mathbf{n} \cdot \frac{1}{2} \cdot
$$

with $\mathrm{n} \cdot \stackrel{\bullet}{. .} \cdot$

This operator has the properties:

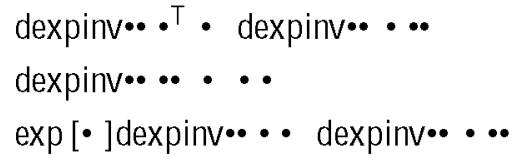

The configuration space of the rigid dumbbell with a fixed point is the unit sphere $S^{2}$, another nonlinear manifold. In the analysis of Sects. 5 and 6 we will make use of the identification of $S^{2}$ with the quotient space $S O(3) \cdot S^{1}$. If $\bullet \mathbf{e}_{2} \cdot \mathbf{e}_{2} \mathbf{e}_{3} \bullet$ is a fixed orthonormal base of $\cdot{ }^{3}$, then for each $\mathbf{d} \cdot \mathrm{S}^{2}$ there exists a unique rotation - - SO(3) that maps the unit vector $\mathbf{e}_{3}$ to $\mathbf{d}$ without drill, i.e.,

$\mathbf{d} \cdot \mathbf{e}_{3} \cdot \cdot \exp \cdot \cdot \cdot \cdot \mathbf{e}_{3} \cdot 0 \cdot$

Only two components of $\cdot$ determine uniquely $\cdot$. A close form expression for such a rotation is

$$
\cdot \cdot d \cdot e_{3} \cdot I \cdot d \cdot e_{3} \cdot \frac{1}{1 \cdot d \cdot e_{3}} \cdot d \cdot e_{3} \cdot \cdot d \cdot e_{3} \cdot \cdot
$$

The time derivative of any unit vector function can thus be written alternatively as

$$
\text { d } \cdot \bullet^{\bullet} \mathrm{e}_{3} \cdot \cdots \cdot \mathrm{e}_{\mathrm{e}} \cdot
$$

for some body angular velocity $\cdot$, orthogonal to $\mathbf{e}_{3}$.

\section{Lagrangian formulation of the free rigid body}

The derivation of the free rigid body equations from the Lagrangian formalism can be found, Next, we outline this process and summarize the main results.
In Sect. 4 we will mimic the same procedure in a discrete setting to derive an integrator.

We consider a rigid body with a fixed point $\mathrm{O} \cdot \boldsymbol{}^{3}$. At this same point we choose a fixed frame of reference with basis vectors $\mathbf{e}_{\mathrm{i}} \cdot \mathrm{i} \cdot \mathbf{1} \cdot \mathbf{2} \cdot 3$, and a moving reference frame $\mathrm{E}_{\mathrm{i}} \cdot \mathrm{i} \cdot \mathbf{1} \cdot \mathbf{2} \cdot 3$ aligned at all times with the principal directions of inertia. The principal moments of inertia are denoted $I_{1} \cdot I_{2} \cdot I_{3}$ and thus, in the moving basis, the inertia tensor is $\mid \cdot \operatorname{diag} \bullet I_{1} \bullet I_{2} \bullet I_{3}$.

The configuration space for this mechanical system is $\mathrm{SO}(3)$. A rotation - - SO(3) represents a configuration of the body, mapping isometrically vectors in a reference configuration to the current configuration. The whole motion of the body is thus represented by a map $\bullet \bullet \cdot \bullet$ providing the configuration at each time instant $\mathrm{t} \cdot \bullet \cdot \bullet \cdot \cdot$. The Lagrangian for the rigid body is a function $L \cdot \cdots \cdot \bullet \bullet$ equal to its kinetic energy. The action functional is defined as:

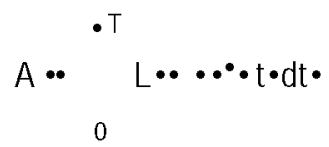

The Euler-Lagrange equations of motion can be derived by invoking Hamilton's principle of stationary action

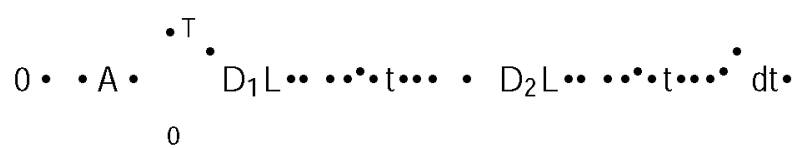

restricting the admissible motions to those with fixed values $\cdots 0 \cdots 0_{0} \cdots \boldsymbol{T}^{-\cdots}$, and integrating by parts. This procedure, which is trivial when the configuration space is linear, becomes somewhat complicated because the variations .. must be of the form (6) and the Lagrangian of the free rigid body has a cumbersome expression as a function of $\bullet$ and $\bullet^{\bullet}$. There is, however, a simpler way of obtaining the equations of motion for a free rigid body. Due to the symmetries of the mechanical system, the Lagrangian of can be reduced to a function - defined on infinitesimal rotations:

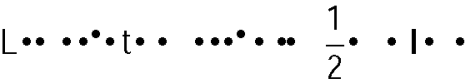

where the (body) angular velocity is defined in Eq. (5). A reduced action can hence be defined as a functional of the reduced Lagrangian $\bullet$

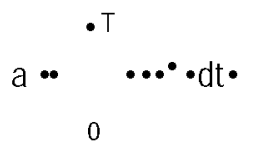

and the reduced Euler-Poincare equations of motion can be obtained from the variational principle:

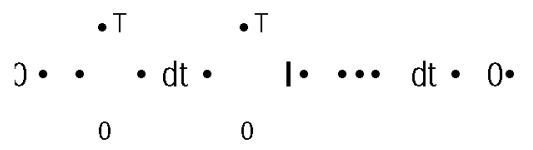


The variations $\cdots$ are not arbitrary functions but, according to Eqs. (5) and (6), must be of the form $\cdots \cdots \cdots \cdot$ for any function $\cdots$ of $t$. Inserting this expression in (18) and integrating by parts we obtain Euler's equations for the rigid body:

$1 \cdot \bullet \cdot \cdots \cdot \cdot$

The free rigid body is a conservative system and thus its total energy, which has the same value as the Lagrangian, is preserved during the motion.

It should be noted that the solution of Eqs. (19) provides the angular velocity $\cdot \bullet \bullet \cdot$ in the time interval of interest, but does not give the motion itself. To obtain the configurations - •t• we must reconstruct them from Eq. (5).

Defining the body and spatial angular momenta, respectively as • • $1 \cdot$ and $\cdots \cdots$, it is straightforward to see that Eq. (19) can be written as

- 0 -

From this point of view, Euler's equations are simply the statement that the spatial angular momentum is preserved.

\section{Variational integrators for the rigid body}

Variational integrators for the rigid body are derived almost in the same way as Euler's equations. First we postulate a discrete Lagrangian and, from it, we obtain a discrete action sum. Both must approximate in a certain way the true Lagrangian and action, respectively. From this point, we simply find the discrete solutions that make stationary the discrete action. In the case of the rigid body this step is not as trivial as with other mechanical systems.

Before defining such functionals we describe the discretization of the time interval in which we want to approximate the motion. For this, let $\mathrm{T} \cdot \bullet_{0} \cdot \mathrm{t}_{1} \cdots \cdots \mathrm{t}_{\mathrm{N}} \cdot$ be $\mathrm{N} \cdot 1$ points in the time interval $\bullet 0 \cdot T \cdot$ such that $0 \cdot t_{0} \cdot t_{1} \cdot$ $\cdots t_{N} \cdot T$. The discrete motion of the rigid body will consist of the rotations at each of these instants, which will be denoted $R \cdot \cdots{ }_{0} \cdot{ }_{1} \cdots \cdots, N$ \} . By means of this discretization, instead of dealing with a differentiable function $\cdots+\cdots 0 \cdot T \cdot S O(3)$ defined on an interval of the real line, we will now be able to work with a finite sequence of rotations $\mathrm{R} \cdot \mathrm{T} \cdot \mathrm{SO}(3)$.

\subsection{The discrete Lagrangian and action sum}

The crucial step in the formulation of variational integrators is the definition of a discrete Lagrangian. This function must approximate the integral of the continuous Lagrangian (16) over a time interval defined by two consecutive time instants in T. Moreover, its explicit expression can only employ data from $\mathrm{R}$.
We pretend to construct the simplest variational integrator for the rigid body. For that, given $t_{n} \bullet t_{n} \bullet 1 \cdot T$, we could define the discrete Lagrangian and discrete action sum as

$$
\begin{aligned}
& L_{d} \cdot n_{n} \cdot n_{n} \cdot 1 \cdot t_{n} \cdot t_{n} \cdot 1 \cdot \underbrace{t_{n} \cdot 1}_{t_{n}} L \cdot \cdot \cdot t \cdot \cdots \cdot \cdot \cdot \cdot \cdot t \cdot d t \cdot \\
& A_{d} \cdot \underbrace{\| \cdot 1}_{n \bullet 0} L_{d} \cdot n_{n} \cdots n \cdot 1 \cdot t_{n} \cdot t_{n} \cdot 1 \cdot \bullet
\end{aligned}
$$

The discrete motion could now be obtained as the sequence $\mathrm{R}$ that makes $\mathrm{A}_{d}$ stationary, under the restrictions that $\cdot$ 。 and ${ }_{N}$ remain fixed.

However, as explained in Sect. 3, the expression of the Lagrangian of the rigid body as a function of the motion $\cdot \cdot \boldsymbol{t}$ and its time derivative is cumbersome. Thus we take a simpler alternative which consists in constructing a discrete reduced Lagrangian and obtaining the discrete Euler-Poincaré equations. The discrete reduced Lagrangian $\cdot d$ and corresponding action $\mathrm{a}_{\mathrm{d}}$ should approximate the reduced Lagrangian and action, respectively, and hence:

$$
\begin{aligned}
& \cdot d \cdot{ }_{n \cdot 1}^{n} \cdot t_{n} \cdot t_{n} \cdot 1 \cdot t_{t_{n}}^{b_{n} \cdot 1} \cdots \cdot \cdot \cdot \cdot t \cdot d t \cdot \\
& a_{d} \cdot{ }_{n \cdot 0}^{\bullet \cdot 1} \cdot d \cdot n_{n} \cdot 1 \cdot t_{n} \cdot t_{n} \cdot 1 \cdots
\end{aligned}
$$

A simple expression for the discrete reduced Lagrangian is:

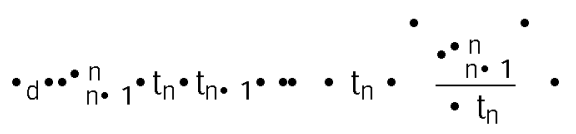

where $\cdot t_{n} \cdot t_{n \cdot 1} \cdot t_{n}$ and $\cdot{ }_{n \cdot 1}^{n}$ has the geometric interpretation of being the incremental rotation vector from ${ }^{n}$ n to $\bullet \cdot 1$. The motivation behind this definition is as follows. To approximate an integral of the reduced Lagrangian $\bullet$, we use the reduced Lagrangian evaluated with an approximation to the continuous angular velocity. The employed vector is the exact angular velocity of the smoothest curve connecting - $n$ and ${ }_{n} \bullet{ }_{1}$, the so-called spherical interpolation between these two rotations The midpoint approximation of the Lagrangian gives a second order approximation of the integral (22) and is responsible for the second order accuracy of the resulting integrator.

In passing from the full variational principle to the reduced problem, the stationary solution that we seek is no longer a sequence of rotations but a sequence of incremental rotation vectors $• \cdot{ }_{1}^{0} \cdot{ }_{2}^{1} \ldots \ldots N_{N}^{N} \cdot 1$. Given the initial rotation ${ }_{0}$, the sequence $\cdot$ can be employed to reconstruct the full motion $\mathrm{R}$. 


\subsection{The discrete equations of motion}

In Sect. 3, the equations of motion of the rigid body were obtained from a variational principle which sought the stationarity of the reduced action with respect to a special type of velocity variations. Repeating this same argument now on the action sum (22) and discrete reduced Lagrangian (23) we get:

$$
\begin{aligned}
& 0 \cdot \cdot a_{d} \\
& n_{n \cdot 0}^{\infty \cdot 1 \ldots d \cdot n_{n \cdot 1} \cdot t_{n} \cdot t_{n} \cdot 1} \cdots \frac{n}{n \cdot 1} \\
& \frac{1}{n \cdot 0} \cdot t_{n} \cdot{ }_{n \cdot 1}^{n} \cdots n_{n \cdot 1}^{n} \cdot
\end{aligned}
$$

The variations of the incremental vector are not arbitrary because they must be related to the variations of the configurations in $\mathrm{R}$. Taking variations on the relation (4), using the definition of the operators dexp... and its inverse, we readily get:

$$
\cdots{ }_{n \cdot 1}^{n} \cdot \operatorname{dexpinv} \cdot{ }_{n \cdot 1}^{n} \cdot \cdots n_{n} \cdot R_{n}^{n} \cdot{ }_{1} \cdot{ }_{n} \cdot 1 \cdot
$$

Furthermore, since ${ }_{0}$ o and ${ } \mathrm{N}$ are to be considered fixed in Hamilton's principle, their variations $\boldsymbol{\cdots}_{0} \cdots_{\mathrm{N}}$ must be identically zero. Finally, replacing the expression for the rotation vector variations in (24) we obtain the main result, an expression for the integration algorithm:

Theorem 1 The discrete Euler-Poincaré equations for discrete Lagrangian (23) and action sum (22) are:

$$
\text { dexpinv } \bullet \cdot{ }_{n}^{n} \cdot 1 \cdot 1 \frac{{ }_{n}^{n} \cdot 1}{\bullet t_{n}} \cdot \operatorname{dexpinv} \cdot{ }_{n}^{n} \cdot 1 \cdot 1 \frac{{ }_{n}^{n} \cdot 1}{\cdot t_{n} \cdot 1} \cdot
$$

Proof From the variational statement (24) and the expression for the variations (25) we can write:

$$
\begin{aligned}
& 0 \cdot{ }_{n \cdot 0}^{d \cdot 1} \frac{1}{\cdot t_{n}} l \cdot \frac{n}{n \cdot 1} \cdot \operatorname{dexpinv} \cdot \frac{n}{n \cdot 1} \cdot \cdots n
\end{aligned}
$$

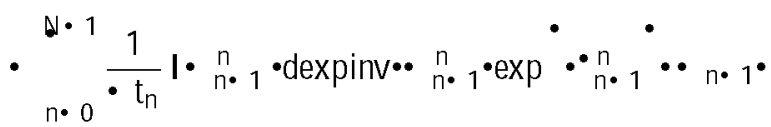

In the first sum we use the fact that $\boldsymbol{\cdot}_{0} \cdot \mathbf{0}$. In the second sum, we shift the sum index $n \cdot n \cdot 1$ and use $\cdot N \cdot \mathbf{0}$ to write:

$$
\begin{aligned}
& 0 \cdot{ }_{n \cdot 1}^{d \cdot 1} \frac{1}{\cdot t_{n}} l \cdot \frac{n}{n \cdot 1} \cdot \operatorname{dexpinv} \cdot{ }_{n}^{n} \cdot 1 \cdots n \\
& \underbrace{\infty \cdot 1}_{n \cdot 1} \frac{1}{\cdot t_{n} \cdot 1} \mid \cdot{ }_{n}^{n \cdot 1} \cdot \operatorname{dexpinv} \cdot{ }_{n}^{n \bullet 1} \cdot \\
& \cdot \exp \cdot \bullet_{n} \cdot 1 \cdot{ }_{n} \cdot
\end{aligned}
$$

Collecting both sums the result reads:

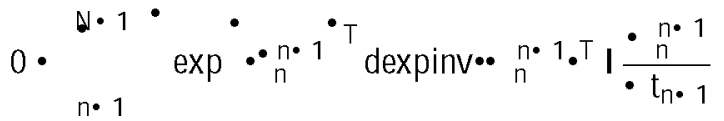

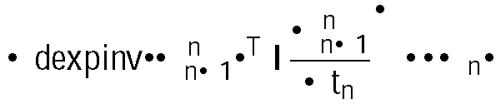

Since the variations $\cdot \mathrm{n}$ are arbitrary for $0 \cdot \mathrm{n} \cdot \mathrm{N}$, the expression inside the parenthesis must vanish and, using relations (10), equation (26) follows

The discrete Euler-Poincaré equations of Theorem 1 provide an update map ${ }_{n}^{n}{ }_{n} 1$ • ${ }_{n}^{n} \cdot 1$. In other words, this map is the integration algorithm that provides the discrete time evolution of the incremental rotations, i.e, the sequence - . As in the continuous case, the solution to the Euler-Poincaré equations does not directly provide the configurations, which must be reconstructed from the incremental rotations: given the initial configuration ${ }_{0}$ and the sequence $\bullet$, the rotation - $n \cdot 1$ is obtained recursively from the previous rotation using the formula ${ }_{n} \cdot 1 \cdot{ }_{n} \exp \cdot{ }_{n} n_{1}$.

We observe that the update equations for the variational integrator are implicit and nonlinear, in contrast with other variational integrators for discrete systems of springs and mass points, or even nonlinear elasticity (see [13]). Only if the three principal moments of inertia are identical, the Algorithm (26) becomes explicit after using property (10).

Remark 1 The initial data of a rigid body problem includes an initial position ${ }_{0} \circ$ and angular velocity ${ }_{0}$ obut what is actually required to start the algorithm is the incremental rotation vector between two consecutive time instants. To obtain such a vector from the available data, the following starting procedure is proposed:

1. Compute from (19) the initial angular acceleration as $\bullet_{0} \cdot I^{\bullet} 1_{0} I_{0_{0}} \cdot \bullet_{0} \cdot$

2. Approximate the angular velocity at time $t \cdot \cdot \cdot \frac{\boldsymbol{t}_{0}}{2}$ by

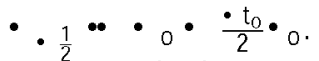

3. Compute the incremental rotation vector between time $\mathrm{t} \cdot \cdots \mathrm{t}_{0}$ and time $\mathrm{t} \cdot 0$ by $\cdot{ }_{0}^{1} \cdot \mathrm{t}_{0} \cdot \frac{1}{2}$.

We note that the vector ${ }_{0}{ }_{0}$ is the angular velocity at the time instant $t \cdot 0$, but the velocity $\cdot \dot{0}^{1} \cdot t_{0}$ must be equal to the average angular velocity during the time interval $\cdot \bullet \mathrm{t}_{0} \bullet \boldsymbol{0} \cdot$ Choosing simply $\cdot{ }_{0}^{1} \cdot \bullet_{0} \cdot t_{0}$ will spoil the second order accuracy of the method. In fact, any other second order method could be employed to obtain the initial incremental rotation, for the variational properties of the attendant method are independent of it.

\subsection{Conservation laws}

We have seen in Sect. 3 that the motion of the free rigid body preserves the spatial angular momentum. As Noether's 
theorem shows, this is a consequence of the symmetry of the Lagrangian with respect to (left) superposed rotations.

The discrete Lagrangian is also invariant with respect to the left action of the rotation group. The discrete Noether's theorem guarantees the existence of a corresponding conservation law in the discrete solution, the conservation of discrete angular momentum. The precise form of this quantity is obtained from the stationarity of the discrete action itself under superposed rotations but can be obtained directly from the discrete equations of motion (26). For this, recall that Euler's equations can be understood as the statement of preservation of spatial angular momentum (see Eq. (20)). In order to show that the algorithmic equation (26) expresses a similar conservation principle, we note that the latter can be written as

$$
\begin{aligned}
& \cdot{ }_{n} \operatorname{dexpinv} \cdot \cdot{ }_{n}{ }_{n} \cdot 1 \cdot \frac{\bullet{ }_{n}^{n} \cdot 1}{\cdot t_{n}}
\end{aligned}
$$

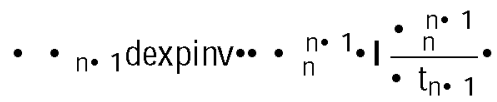

or more compactly as

$\cdot{ }_{n}^{n} 1_{1} \cdot n_{n}^{n} 1$

if the following notation is introduced:

${ }_{\mathrm{n}}^{\mathrm{n}} \mathbf{1}_{1} \cdot \mathrm{n}_{\mathrm{n}} \cdot \mathrm{n}_{\mathrm{n}}^{\mathrm{n}} \mathbf{1}^{\circ}$

$\cdot{ }_{n}^{n} 1_{1} \cdot \operatorname{dexpinv} \cdot \cdots \cdot n_{n}^{n} \cdot 1 \cdot \frac{n}{n} \cdot 1 \cdot$

$\cdot{ }_{n \cdot 1}^{n} \cdot \frac{{ }_{n}^{n} \cdot 1}{\cdot t_{n}} \cdot$

The vectors ${ }_{n} n_{1} \cdot{ }_{n \cdot 1}^{n}$, and $\bullet{ }_{n} \cdot 1$ are, respectively, discrete approximations to the average spatial angular momentum, body angular momentum, and body angular velocity in the interval $\bullet t_{n} \cdot t_{n} \cdot 1^{\bullet}$. Moreover, they are second order approximations to their continuum counterparts at time $t \cdot$ $\frac{1}{2} \cdot t_{n} \cdot t_{n} \cdot 1^{\bullet}$.

As it happens with other variational integrators, the numerical method defined by Eqs. (26) does not preserve the spatial angular momentum which results from a natural discretization of the continuous momentum, which we denote ${ }^{-} \cdot \frac{1}{2}$, the momentum at the midpoint, and which for this case would be:

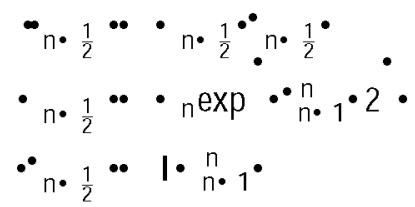

Instead, the algorithmic spatial momentum ${ }_{n \cdot 1}^{n}{ }_{1}$, the one consistent with the variational structure of the method, is exactly preserved in time. As it will be shown in the numerical simulations of Sect. 7 the values of both quantities are similar, but not identical.
Just as variational integrators preserve discrete momenta, the theory of these methods shows that they exactly preserve a discrete symplectic form . The precise statement of this property in the context of the algorithm discussed in the present work, as well as it proof,

\subsection{Final form of the algorithm}

The variational algorithm can be finally written as: given the initial configuration $\cdot 0$ and body angular momentum - ${ }_{0} \cdot{ }_{0}$ of the body,

1. Compute the initial acceleration $\bullet_{0} \cdot I^{\bullet} \bullet_{0} \cdot \bullet_{0}$ and the starting values for the body angular velocity, body incremental rotation and body angular momentum:
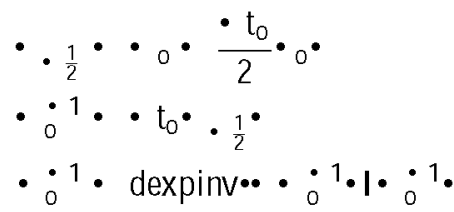

2. Set $n \cdot 0$.

3. Compute the momentum ${ }_{n \cdot 1}^{n} \cdot \exp \cdot \cdots{ }_{n}^{n \cdot 1} \cdot{ }_{n}^{n \cdot 1}$.

4. Solve for the incremental vector ${ }_{n} n_{1}$ in ${ }_{n \cdot 1}^{n}$. dexpinv $\cdot \cdots \cdot{ }_{n \cdot 1}^{n} \cdot 1 \frac{{ }_{n}^{n} \cdot 1}{\cdot t_{n}}$

5. Update the rotation ${ }_{n \cdot 1} \cdot{ }_{n} \exp \cdot \bullet_{n} n_{1}$.

6. Update the angular velocity ${ }_{\mathrm{n} \cdot 1}^{\mathrm{n}} \cdot \frac{\mathrm{n}^{\mathrm{n}} \cdot 1}{\cdot \mathrm{t}_{\mathrm{n}}}$.

7. If $n \cdot N$, increment $n$ and go to 3 .

We note that the body angular velocity, as defined in step 6 , can be eliminated completely from the algorithmic update equations. Its value can be calculated after the whole trajectory is computed either from the values of the incremental rotation vectors or from the body momentum.

\section{Lagrangian formulation of the Dumbbell}

We consider next the dumbbell, another example of a mechanical system with a nonlinear configuration space. In contrast with the free rigid body studied previously, the next example includes forcing.

The dumbbell consists of two point masses $m_{1} \cdot m_{2}$ under external forces, connected by a rigid bar of length $b$. The configuration of such a body is completely determined by the position of its center of mass and the orientation of the rigid bar. In this work we focus on the simpler case in which the center of mass is a fixed point.

The orientation of the bar can be described with a unit vector $\mathbf{d} \cdot{ }^{3}$ pointing from the center of mass towards 
point mass $m_{1}$. The configuration space is thus $S^{2}$, the unit sphere in ${ }^{3}$, a nonlinear two dimensional manifold.

Let $\mathrm{I} \cdot \mathrm{m}_{1} \mathrm{~m}_{2} \mathrm{~b}^{2} \cdot \mathrm{m}_{1} \cdot \mathrm{m}_{2} \cdot$ be the (scalar) inertia of the dumbbell. The Lagrangian of this mechanical system is equal to its kinetic energy minus the potential energy of the external forces, which we assume that depends only on the configuration and can be written alternatively as

$$
\begin{aligned}
& L \cdot \boldsymbol{d} \cdot \mathbf{d} \bullet t \cdot \bullet \frac{1}{2} \mid \cdot \mathbf{d} \cdot \boldsymbol{\bullet}^{2} \cdot V_{\text {ext }} \cdot \mathbf{d} \cdot \bullet \quad \text { or }
\end{aligned}
$$

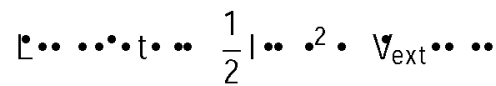

where - is the unique rotation that maps $\mathbf{e}_{3}$ into $\mathbf{d}$ without drill. The action associated with the previous Lagrangian is

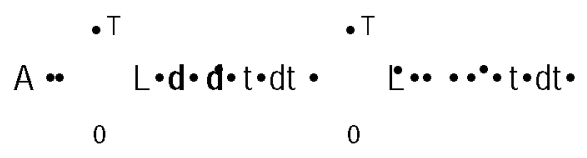

We assume, for simplicity, that the potential of the external forces is of the form $V_{\text {ext }} \cdot \mathbf{d} \cdot \boldsymbol{\bullet} \cdot \mathbf{d} \cdot \mathbf{g}$ for some function of time $\mathbf{g} \cdot \boldsymbol{v} \cdot$ representing the moment of the external forces with respect to the center of mass. Then, the Euler-Lagrange equations of motion can be easily obtained by invoking the stationarity of this action with respect to variations of the director, resulting in:

$I \frac{d}{d t} \cdot d \cdot a \cdot \cdot d \cdot g \cdot$

Equivalently, the Euler-Lagrange equation can be written in terms of the rotation - and its time derivative as:

I.* $\mathbf{e}_{3} \cdot{ }^{\prime}{ }^{\top} \mathbf{g} \cdot \bullet$

In order to investigate the effects of symmetry in the equations, and later in the variational integrator, we assume that the loading $\mathbf{g}$ is of the form $\mathbf{g} \bullet \boldsymbol{t} \cdot \boldsymbol{g} \bullet \bullet \cdot \boldsymbol{e}_{3}$, where $\mathrm{g}$ is a scalar function. In this way, the potential energy is invariant under the action of the subgroup of rotations that leave $\mathbf{e}_{3}$ invariant, which we denote $\mathrm{G}$. Since the kinetic energy is fully $\mathrm{SO}(3)$ invariant, the symmetry group of the Lagrangian will be $\mathrm{G}$.

By Noether's theorem, there is a conserved quantity associated with the symmetry the Lagrangian with respect to the action of $\mathrm{G}$. It can be verified that this quantity is precisely the projection of the angular momentum

- I d $\cdot d \cdot \mid \cdot \cdot$

on the $\mathbf{e}_{3}$ direction.

\section{A variational integrator for the Dumbbell}

To obtain the equations of the variational integrator for the dumbbell we follow the steps taken in Sect. 4 for the rigid body. We consider the same discretization of the integration interval and we look for a sequence of approximations to the dumbbell orientation $\mathrm{D} \cdot \bullet \cdot \mathbf{d}_{0} \cdot \mathbf{d}_{1} \cdots \cdots \mathbf{d}_{\mathrm{N}} \cdot$, or equiva-

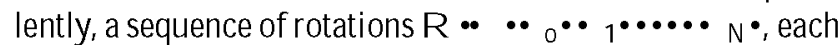
of the latter mapping $\mathbf{e}_{3}$ without drill to the corresponding unit vector. The crucial step in the formulation of the integrator is the choice of the discrete Lagrangian and we select the following trapezoidal approximation:

$$
\begin{aligned}
& \bullet_{d} \cdot{ }_{n} \cdot e_{n} \cdot 1 \cdot t_{n} \bullet t_{n} \cdot 1^{\bullet} \\
& \bullet \frac{1}{\cdot t_{n}} \cdot{ }_{n}^{n} \cdot 1^{2} \cdot \frac{t_{n}}{2} \cdot v_{\text {ext }} \cdot{ }_{n} \cdots v_{\text {ext }} \cdot{ }_{n} \cdot 1_{1} \cdots
\end{aligned}
$$

which for the specific form chosen for the external forces, simplifies to

$$
\begin{aligned}
& \bullet_{d} \cdot{ }_{n} \bullet{ }_{n} \cdot 1 \cdot t_{n} \cdot t_{n} \cdot 1 \cdot \\
& \cdot \frac{1}{\cdot t_{n}} \cdot{ }_{n}^{n} \cdot 1^{n} \cdot \frac{t_{n}}{2} \cdot{ }_{n} \cdot n_{n} \cdot 1^{\bullet} e_{3} \cdot g \cdot
\end{aligned}
$$

In these last two expressions, the incremental rotation vector ${ }_{n}{ }_{n}{ }_{1}$ is such that ${ }_{n} \bullet{ }_{1} \cdot{ }_{n} \exp \cdot{ }_{n}{ }_{n}$, and, in view of (11), it is orthogonal to $\mathbf{e}_{3}$. After defining the discrete action sum as in (21), the discrete equations of motion are readily obtained:

Theorem 2 The discrete equations of motion for the free dumbbell associated with the discrete Lagrangian (41) are:

$$
\text { I } \frac{{ }_{n}^{n} \cdot 1}{\cdot t_{n}} \cdot \frac{n_{n}^{n \cdot 1}}{\cdot t_{n} \cdot 1} \cdot \frac{t_{n} \cdot \cdot t_{n} \cdot 1}{2} e_{3} \cdot \cdots{ }_{n}^{\top} \mathbf{g} \cdot \cdot
$$

with, the rotation update being, as above, ${ }_{n} \cdot 1 \cdot{ }_{n}$ $\exp \cdot{ }_{\mathrm{n}}^{\mathrm{n}} 1$.

Proof The result is proved by imposing the stationarity of the discrete action sum with respect to variations of the form:

$\bullet_{n} \cdot{ }_{n} \cdot{ }_{n} \cdot \quad$ with $\cdots{ }_{n} \cdot e_{3} \cdot 0 \cdot$

The steps are very similar to those in the proof of Theorem 1. Details are omitted.

\subsection{Conservation laws}

The discrete Lagrangian (40) inherits the symmetry of the continuous one, hence it is invariant under the action of $\mathrm{G}$ that tranşforms every unit vector of the sequence $D$ as $\mathbf{d}_{n}$. $\exp \cdot \mathbf{e}_{3} \mathbf{d}_{n}$, for some $\cdot \cdot \cdot$. By the discrete version of Noether's theorem, a conserved quantity must exist. It can be identified as the projection onto the $\mathbf{e}_{3}$ direction of the discrete momentum

$$
\cdot{ }_{n \cdot 1}^{n} \cdot \frac{\dot{ }_{n} \cdot n_{n} \cdot 1}{2} \cdot{ }_{n \cdot 1}^{n} \cdot \quad \cdot{ }_{n \cdot 1}^{n} \cdot \frac{n_{n}^{n} \cdot 1}{\cdot t_{n}} \cdot
$$

In this expression, ${ }_{n} n_{1}$ is the algorithmic approximation of the body angular velocity in the interval $\bullet_{n} \cdot t_{n} \bullet 1^{\bullet}$. To show that the third component of $\cdot{ }_{n \cdot 1}^{n}$ is preserved, we take the 
dot product of both sides of (42). Noting that the right hand side vanishes for $\mathbf{g} \cdot \mathrm{g} \cdot \mathbf{t} \cdot \mathbf{e}_{3}$ it follows that

$l \cdot n \frac{n_{n}^{n} 1}{\cdot t_{n}} \cdot e_{3} \cdot l \cdot n \frac{n_{n}^{n \cdot 1}}{t_{n} \cdot 1} \cdot e_{3} \cdot$

Finally, using the properties of the exponential map, we can write the previous expression also as:

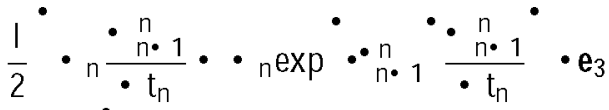

$$
\begin{aligned}
& \cdot \frac{1}{2} \cdot \frac{n_{n}^{n} 1}{n \cdot t_{n} 1} \cdot{ }_{n} \exp \bullet \cdot n_{n \cdot 1} \cdot \frac{n_{n}^{n} 1}{t_{n} \cdot 1} \cdot e_{3} \bullet
\end{aligned}
$$

and, using the definition of the incremental rotation vector, we conclude that

$$
\begin{aligned}
& \frac{1}{2} \cdot n \frac{n_{n} \cdot 1}{\cdot t_{n}} \cdot n \cdot 1 \frac{n_{n} \cdot 1}{t_{n}} \cdot e_{3} \\
& \cdot \frac{1^{\bullet}}{2} \cdot \frac{n^{n} n^{n}}{t_{n \cdot 1}} \cdot n \cdot 1 \frac{n_{n}^{n \cdot 1}}{t_{n \cdot 1}} \cdot e_{3} \cdot
\end{aligned}
$$

which is precisely the statement of conservation of discrete angular momentum.

Similarly to what happened in the case of the rigid body, the variational integrator (42) possesses a discrete conservation law associated with the continuous one, but not identical . In this second example, the most natural definition of angular momentum would be:

$\cdot{ }_{n} \cdot \frac{1}{2} \cdot \cdots \cdot{ }_{n} \cdot \frac{1}{2} \cdot{ }_{n}^{n} \cdot 1 \cdot$

with $\bullet n_{\frac{1}{2}}$ being the rotation at the midpoint $t_{n} \cdot \frac{1}{2}$ defined as in (33). We conclude by noting that, due to its variational nature, the algorithm (42) preserves a discrete symplectic form.

\subsection{Final form of the algorithm}

The variational method for the dumbbell is a one-step, explicit method. Given the initial unit vector $\mathbf{d}_{0}$ and body angular velocity ${ }_{{ }_{0}}$ of the body, with ${ }_{0} \cdot{ }^{1} \mathbf{e}_{1} \cdot{ }^{2} \mathbf{e}_{2}$,

1. Compute the unique rotation $\cdot$ o that maps $\mathbf{e}_{3}$ to $\mathbf{d}_{0}$ without drill.

2. Compute the initial angular acceleration and use it to obtain the algorithmic angular velocity $\cdot:_{0}^{1}$ in the interval $\cdots \cdot \mathrm{t}_{0} \cdot 0 \cdot$, and the corresponding incremental rotation vector

$$
\begin{aligned}
& \left.\cdot{ }_{0} \cdot\right|^{1} \mathrm{e}_{3} \cdot \bullet_{\mathrm{o}}^{\mathrm{T}} \mathrm{g} \cdot \cdot \\
& \dot{0}^{1} \cdot \bullet_{0} \cdot \frac{\mathrm{t}_{0}}{2} \cdot{ }^{\bullet} \\
& \dot{0}^{1} \cdot \dot{0}^{1} \cdot \mathrm{t}_{0}
\end{aligned}
$$

3. Set $n \cdot 0$.
4. Find the next incremental rotation vector by solving the discrete Euler-Lagrange equation:

$$
\begin{aligned}
& { }_{n \cdot 1}^{n} \cdot{ }_{n}^{n \bullet 1} \cdot \frac{t_{n} \cdot t_{n} \cdot 1}{2 I} \cdots{ }_{n}^{T} \mathbf{g} \bullet \mathbf{e}_{3} \bullet \\
& \cdot \frac{n}{n \cdot 1} \cdot t_{n} \cdot \frac{n}{n \cdot 1} \cdot
\end{aligned}
$$

5. Update the rotation and the direction vector at time $t_{n} \cdot 1$

$$
\cdot n_{1} \cdot{ }_{n} \exp \cdot{ }_{n \cdot 1} \cdot d_{n \cdot 1} \cdot n_{n} \cdot e_{3} \cdot
$$

6. Update the discrete momentum

$$
\cdot{ }_{n \cdot 1}^{n} \cdot \frac{1}{n \cdot n \cdot n} \cdot \frac{n}{n} \cdot 1 \cdot
$$

7. If $n \cdot N$, increment $n$ and go to 3 .

We note that the angular velocity, the momentum, and the direction vector could be eliminated from the algorithm equations and postprocessed from the solution later. Only the configuration variables (the rotations) need to be stored.

\section{Numerical simulations}

7.1 The free rigid body

In this section we present numerical results of the motion of a rigid body of (non-physical) inertia tensor I $\operatorname{diag} \cdot 9 \cdot 4 \cdot 1 \bullet$ The initial position of the body is given by the matrix

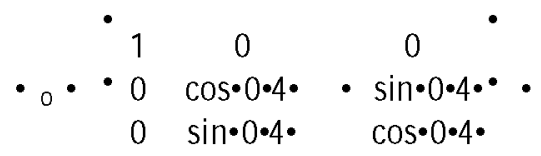

and it has initial angular velocity ${ }_{0} \cdot 3 \mathrm{E}_{1} \cdot 12 \mathrm{E}_{3}$.

First we evaluate the qualitative properties of the proposed variational algorithm in the reduced picture. We study the solution of the described rigid body in the time interval $\bullet \cdot \cdot 5 \cdot$ with a time step size of $\bullet t \cdot 0 \cdot 05$.

Figure 1 shows the evolution of the three components of the algorithmic body momentum ${ }_{n}{ }_{n} \cdot 1$ and the standard $\cdot{ }_{\mathrm{n}} \frac{1}{2}$. The curves on the plots are almost indistinguishable but only the norm of the first momentum is exactly preserved. The norms of the discrete and "standard" momenta are shown on the left of Fig. 2. Whereas the norm of the discrete momentum is exactly preserved, the norm of the "standard" momentum oscillates. The total energy in the solution provided by the variational integrator fluctuates around a constant value, as it is shown in Fig. 2 (bottom). As with other variational integrators, the average (discrete) energy value is not the exact one but close to it. See, for example, [27]. Of course, 

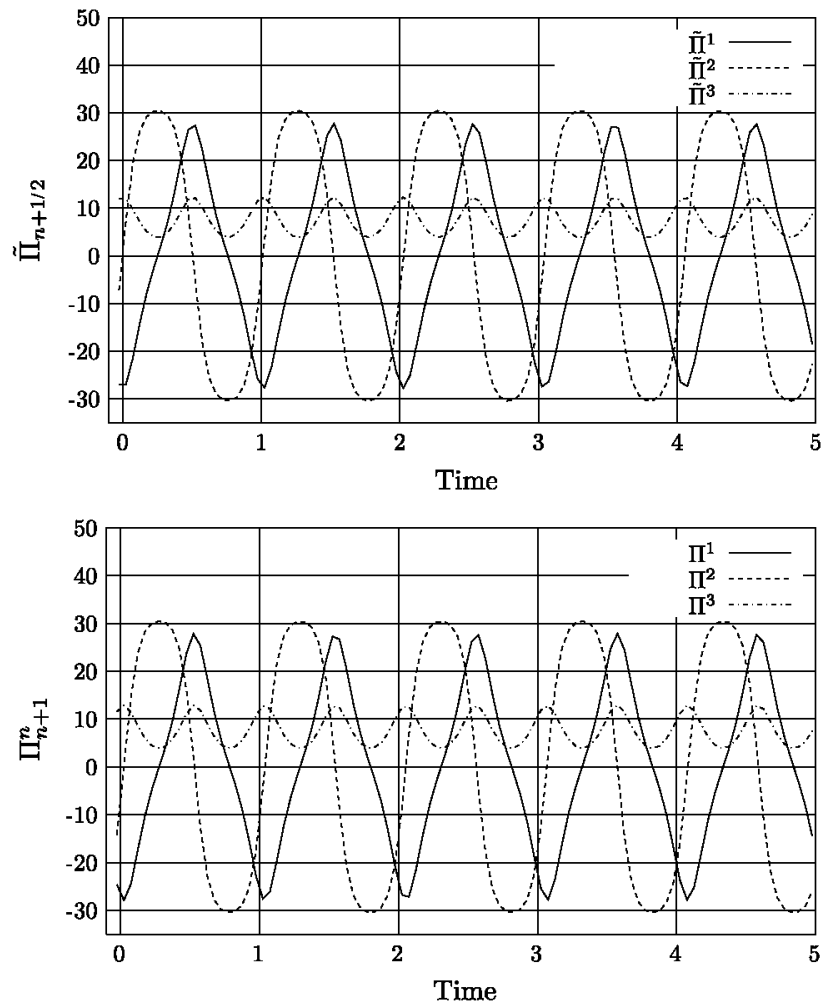

Fig. 1 Time evolution of the components of the "standard" body angular momentum $\bullet_{n} \cdot \frac{1}{2}$ (left) and the variationally consistent discrete angular momentum $\bullet{ }_{n} \cdot 1$ (right)

as the time step is refined, the average value of the discrete energy converges to the exact value.

Next we explore the accuracy of the solution provided by the variational integrator. For the problem under study, an analytical solution can be found for the exact body momentum $e_{\text {ex }} t^{\bullet}$

This allows to precisely measure the errors made in the solution of Euler equations. Figure 3 shows the errors made in the time integration of Euler equations with the proposed integrator and three other second order methods. The first method is the midpoint rule,

The second one is the energy-momentum The last one is the Liemid integrator

All these methods are described in the Appendix. The first two preserve energy and momentum during the motion. The last one preserves the momentum but the energy, which is not preserved, oscillates around a constant value, similarly to the proposed method.

Figure 3 clearly shows the second order accuracy of all the methods compared. It is also apparent that, for this example, errors are smaller in the case of the canonical midpoint rule.

Also, we compare the accuracy in the computation of the configuration (rotation) of all these methods. For that, we compute a reference solution with the midpoint rule and a time step one order of magnitude smaller that the smallest of
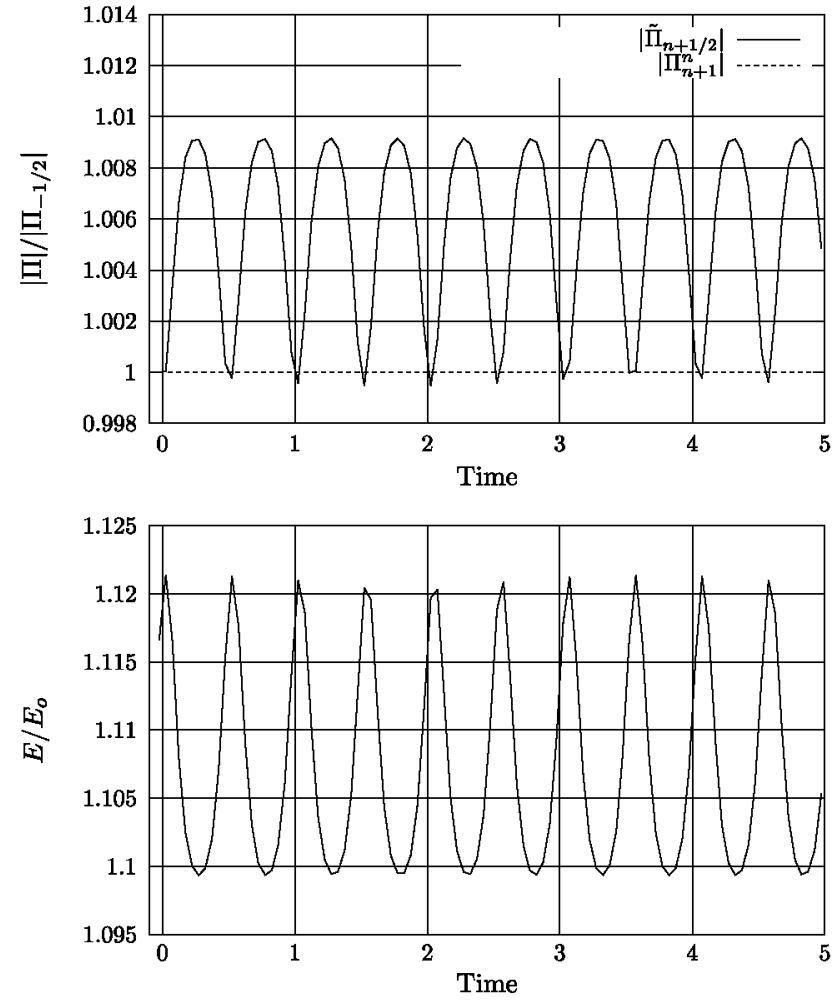

Fig. 2 Norm of the "standard" body angular momentum $\bullet_{\mathrm{n}}{ } \frac{1}{2}$ (left) and total energy (right). Both quantities are divided by the corresponding values of the exact solution
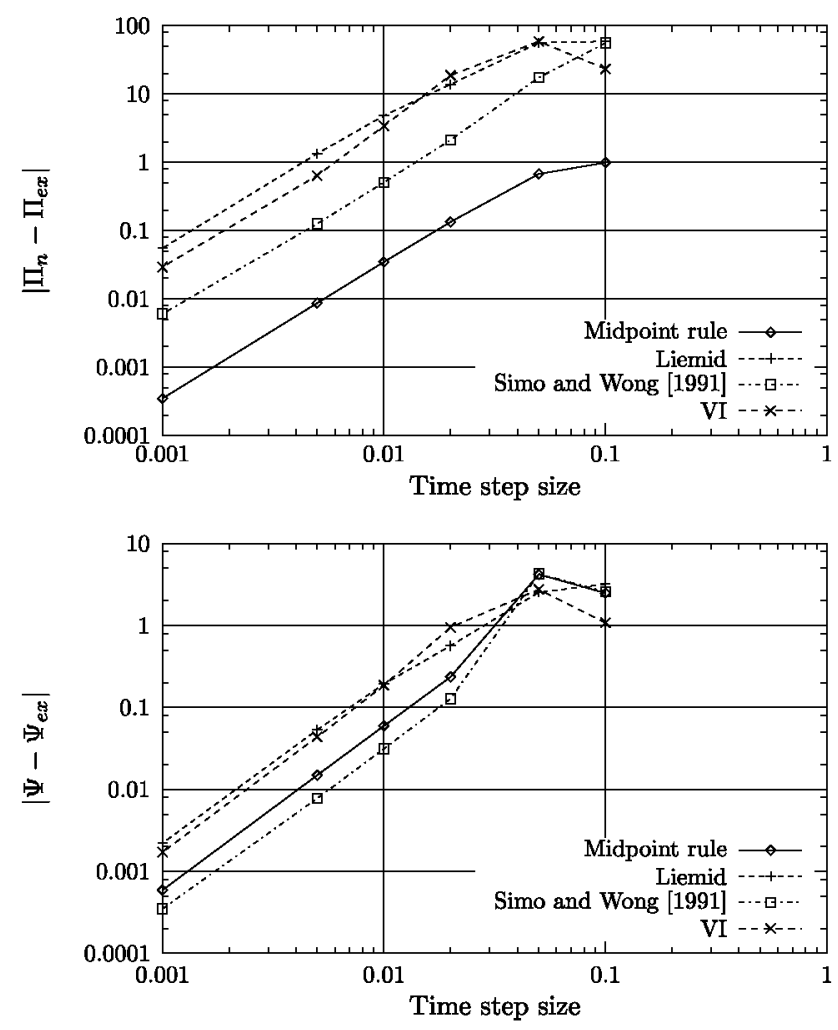

Fig. 3 Errors in the body angular momentum (left) and total rotation vector (right) at the end of the integration interval 
the compared trajectories. For each of the compared methods and the reference solution, the rotation vector - associated with the rotation at time $T$ - 5 is calculated. On the right of Fig. 3 the errors of these vectors are depicted. In this case, the most accurate solution is provided by the energy-momentum method.

Recently, it has been pointed

that conserving algorithms such as the ones of references $[4,23]$ are not as accurate as expected in long term simulations of problems involving forcing. It remains to investigate the accuracy in such situations of the corresponding variational integrator. The equations of such method can be obtained from the stationarity of the associated discrete action, but are more involved than (26) and fall outside the scope and goals of the current work.

\subsection{The dumbbell}

In the second example we consider a dumbbell of inertia I 2 under an applied torque $\mathbf{g} \cdot \mathbf{*} \cdot 3 \mathbf{e}_{3}$. The initial orientation vector and angular velocity are

$\mathbf{d}_{0} \cdot \cdots \sin \bullet 0 \cdot 3 \cdot \mathbf{e}_{2} \cdot \cos \bullet 0 \cdot 3 \cdot \mathbf{e}_{3} \bullet \cdot{ }_{0} \cdot \cdots 3 \mathbf{e}_{1} \cdot 2 \mathbf{e}_{2} \bullet$

As in the first example, we first examine the qualitative features of the variational solution in the time interval $\cdot 0 \cdot 5 \cdot$ with a time step size $\cdot t \cdot 0 \cdot 05$.

Figure 4 depicts the evolution of the three components of the discrete angular momentum (44) and the third value of the "standard" momentum $\bullet_{n \cdot 1 \cdot 2}$. The exact preservation of the third component of the discrete angular momentum can be observed. In contrast, the same component in the momentum - ${ }_{n}{ }_{2}^{1}$ oscillates. The total energy in the discrete solution, depicted in Fig. 5, also oscillates but always remains close to initial value (since the system is conservative, this is always the exact value).

Finally, in Fig. 6, the errors in the computation of the angular momentum and the direction vector at the end of the simulation interval are shown, and compared with the results of two other common second order integrators. See Appendix B for a summary of these two algorithms. The solutions obtained with each method are compared with a reference solution obtained with the midpoint rule and a small time step of size $t_{\text {ref }} \cdot 10^{\bullet}$. The figure reveals the second order accuracy of the three methods. We stress the fact that the variational integrator in the only explicit method of the three compared.

\section{Summary}

We have presented the simplest variational integrators for Euler's equations and the equations of motion of the rigid
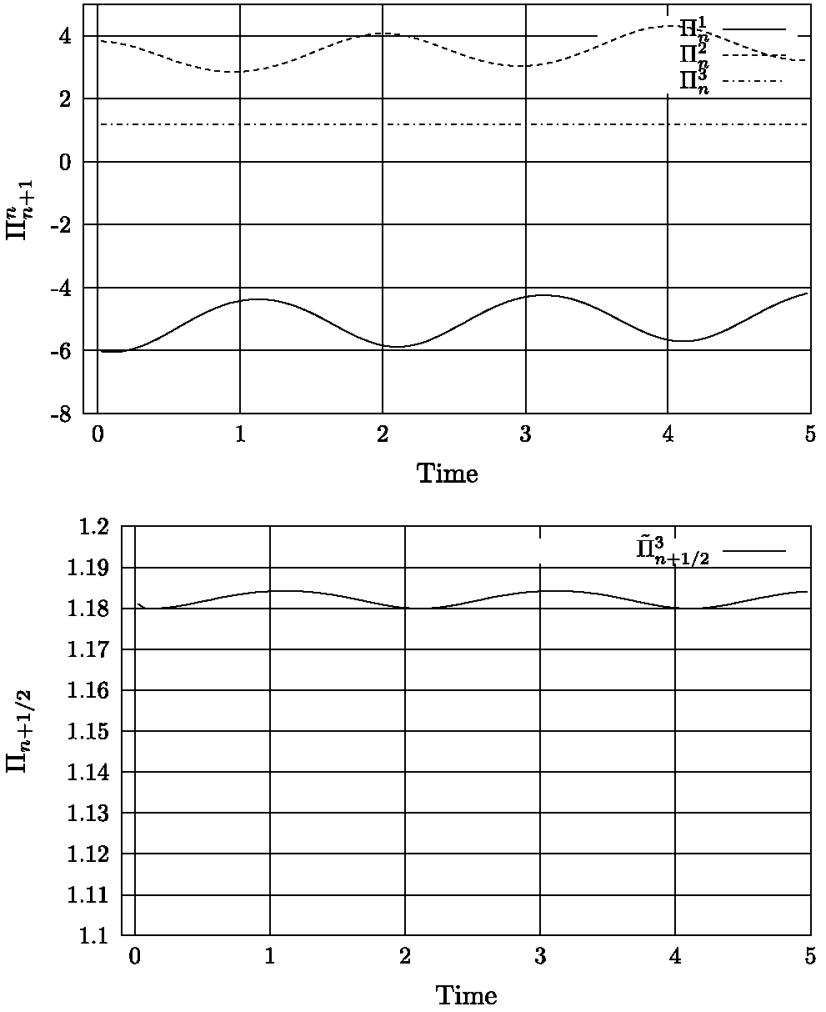

Fig. 4 Time evolution of the three components of the discrete body angular $n_{n}$ (left) and the third component of $\bullet{ }_{n} \cdot 1 \cdot 2$ (note the different scales)

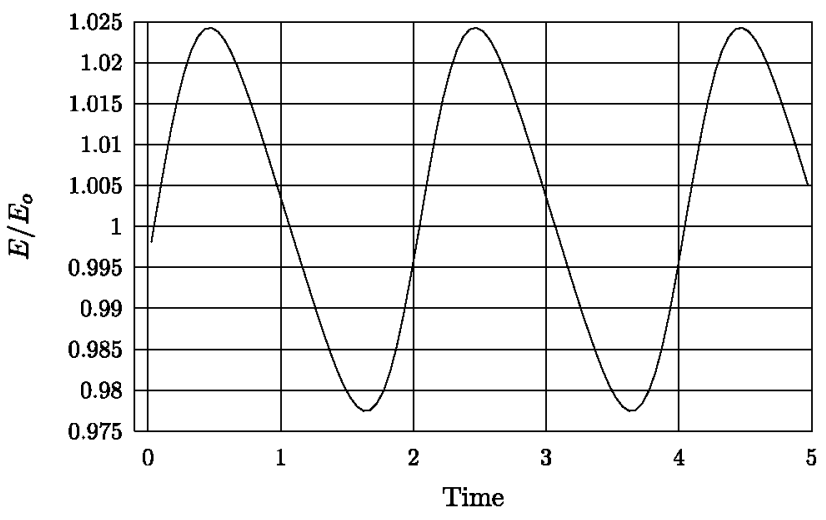

Fig. 5 Normalized value of the total energy

dumbbell. Both integrators are obtained invoking the stationarity of their corresponding action sums, defined in terms of discrete Lagrangians whose specific form has been provided. These Lagrangians are formulated on nonlinear manifolds and do not make use of Lagrange multipliers.

For Euler's equations, the resulting method is a nonlinear, one step, second order accurate, implicit update map with a computational cost comparable to that of similar implicit 

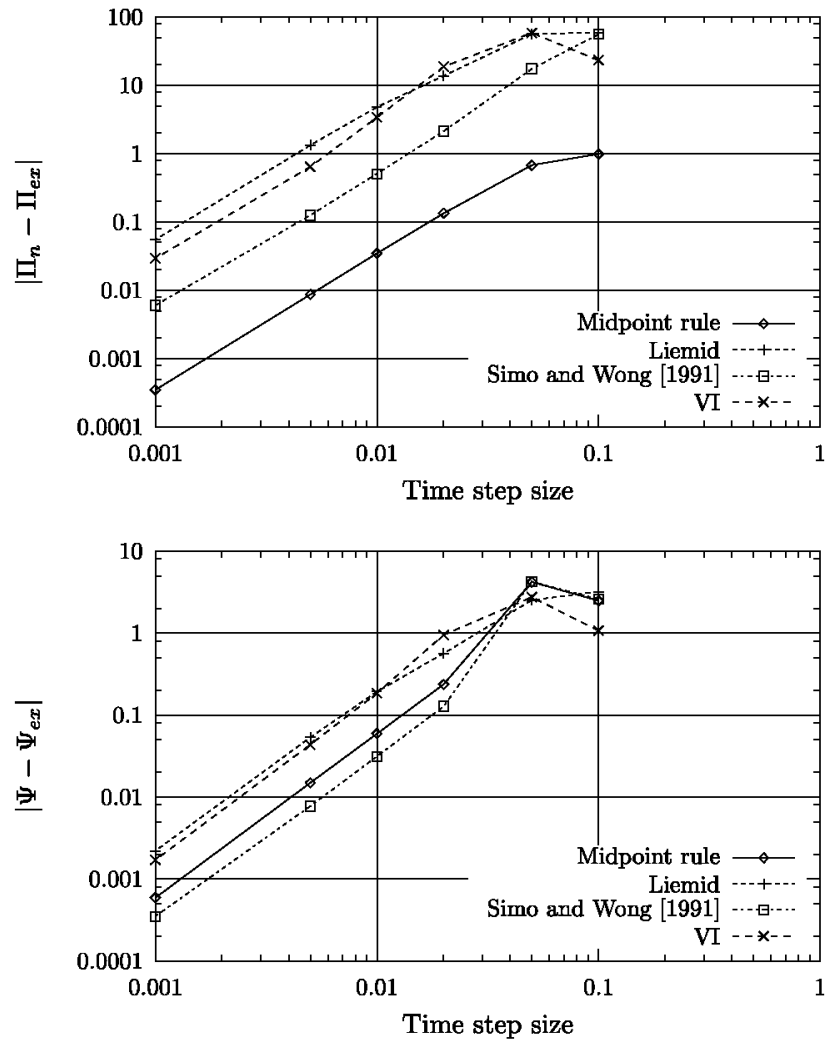

Fig. 6 Errors in the body angular momentum (left) and total rotation vector (right) at the end of the integration interval

methods for Euler's equations. In the case of the dumbbell, the method is second order accurate but explicit. Both methods have interesting geometrical properties, among which is the presence of discrete conservation laws. We have identified the precise form of the discrete momentum for each problem and algorithm studied.

We have presented numerical simulations to illustrate the properties and the accuracy of the proposed methods. The results confirm that the variational methods preserve a discrete momentum and provide a oscillatory energy that closely approximates the exact value of the continuum equations. Numerical tests hast been performed to evaluate the accuracy of the proposed methods and compare them with similar integrators available in the literature. The results suggest that the accuracy of the variational methods is similar to that of other popular second-order methods.

Extensions of the proposed methods to integrate, for example, the equations of the heavy top are not difficult, although the final form of the equations is rather long, as compared with other integrators. In contrast, our attempts to develop higher order variational integrators for the rigid body as well as second order variational methods for the dynamic equations of geometrically exact rods lead us to conclude that both tasks are quite involved.
A Appendix: Summary of integrators for the rigid body equations

\section{A.1 The midpoint rule [4]}

Given ${ }_{0}$ and $\cdot{ }_{0}:$

1. Set $n \cdot 0$.

2. Solve for $\cdot \mathrm{n} \bullet 1$ in

$$
\begin{aligned}
& \mathrm{I} \frac{\mathrm{n} \cdot 1 \cdot{ }^{\mathrm{n}}}{\cdot \mathrm{t}} \cdot \mathrm{I}_{\mathrm{n} \cdot \frac{1}{2}} \cdots \operatorname{\mathrm {n}}_{\frac{1}{2}} \cdot \\
& { }_{n} \cdot \frac{1}{2} \cdot \frac{1}{2} \cdot{ }_{n} \cdot n_{n} \cdot 1 \cdot \bullet
\end{aligned}
$$

3. Update the rotation by $\bullet \mathrm{n} \bullet 1^{\bullet} \cdot \mathrm{n}_{\mathrm{n}}$ cay $\bullet \mathrm{t} \cdot \mathrm{n} \bullet \frac{1}{2} \bullet$, where cay $\cdots$ is the Cayley map defined by:

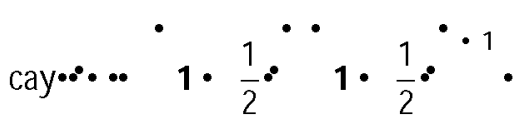

4. If $n \cdot N$, increment $n$ and go to 2 .

A.2 The conserving algorithm of [23]

Given $\cdot{ }_{0}$ and $\cdot{ }_{0}$ :

1. Set $n \cdot 0$.

2. Solve for $\bullet n_{\bullet} 1$ in

$$
\exp \frac{\bullet t_{n}}{2} \cdot \bullet_{n} \cdot \bullet_{n} \cdot 1 \quad l \cdot{ }_{n} \cdot 1 \cdot 1 \cdot n_{n}
$$

3. Compute the incremental rotation vector $\cdot \cdot \frac{\cdot t_{n}}{2} \cdot{ }_{n} \cdot$ - $n \cdot 1^{\bullet}$.

4. Update the rotation by ${ }_{n} \cdot{ }_{1} \cdot{ }_{n} \exp \cdot \bullet$.

5. If $n \cdot N$, increment $n$ and go to 2 .

A.3 The Liemid algorithm of [9]

Given ${ }_{0}$ and $\cdot{ }_{0}$ :

1. Set $n \cdot 0$.

2. Solve for $\cdot$ in

$$
\cdot \cdot \mathrm{t} \mathbf{l}^{\cdot 1} \exp \cdot \frac{1}{2} \cdot \bullet \boldsymbol{l} \cdot \mathrm{n} \cdot
$$

3. Update the rotation by ${ }_{n} \cdot 1_{1} \cdot{ }_{n} \exp \bullet \cdot \bullet$.

4. Update the angular velocity by ${ }_{n} \bullet 1^{\bullet} I^{1} \exp \cdot \cdots$ I. $\mathrm{n}$.

5. If $n \cdot N$, increment $n$ and go to 2 . 


\section{B Summary of integrators for the Dumbbell}

B.1 The midpoint rule in conservation form

Given $\bullet{ }_{o}$ and $\bullet{ }_{0}$ : Given $\bullet$ o and $\bullet$ o:

1. Set $n \cdot 0$.

2. Solve for ${ }_{n} \cdot 1 \cdot \cdots n_{n} \cdot 1$ in

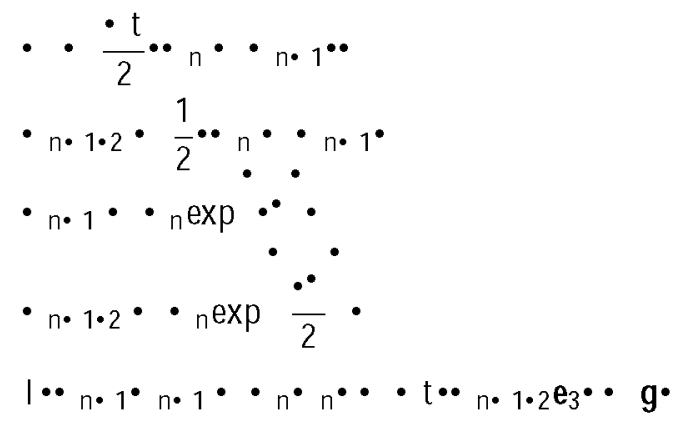

3. If $n \cdot N$, increment $n$ and go to 2 .

B.2 The conserving algorithm of [23]

Given ${ }_{0}$ and $\cdot{ }_{0}$ :

1. Set $n \cdot 0$.

2. Solve for ${ }_{n} \cdot 1 \cdot \cdots{ }_{n} \cdot 1$ in

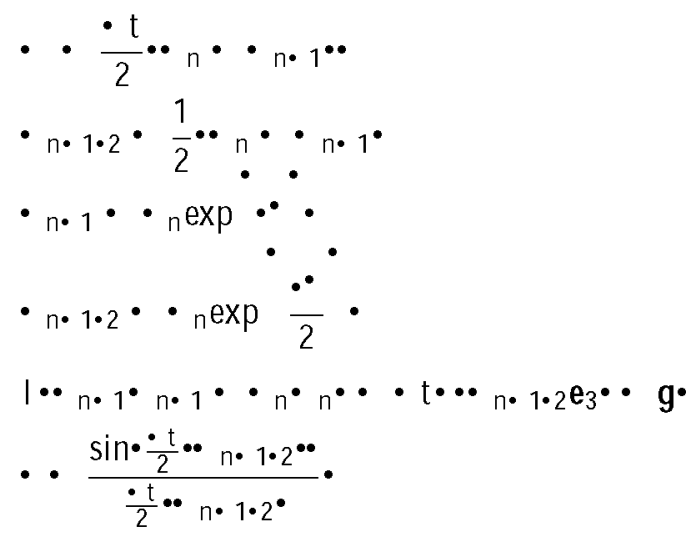

3. If $n \cdot N$, increment $n$ and go to 2 .

\section{References}

Antman SS (1992) Nonlinear problems of elasticity. Springer, Heidelberg

Argyris JH (1982) An excursion into large rotations. Comput Methods Appl Mech Eng 32:85-155
Arnold VI (1989) Mathematical methods of classical mechanics. Springer, Heidelberg

Austin M, Krishnaprasad PS, Wang LS (1993) Almost Poisson integration of rigid body systems. J Comput Phys 107(1):105117

Goldstein H (1980) Classical mechanics, 2nd edn. AddisonWesley, Reading

Hairer E, Lubich C, Wanner G (2002) Geometric numerical integration. Springer series in Computational Mathematics. Springer, Heidelberg

Iserles A, Munthe-Kass HZ, Norsett SP, Zanna A (2000) Lie group methods. Acta Numer 9:215-365

Kharevych L, Weiwei, Tong Y, Kanso E, Marsden JE, Schröder $P_{t}$ Desbrun M (2006) Geometric, variational integrators for computer animation. In: Eurographics/ACM SIGGRAPH symposium on Computer Animation, The Eurographics Association

Krysl P (2005) Explicit momentum-conserving integrator for dynamics of rigid bodies approximating the midpoint lie algorithm. Int J Numer Methods Eng 63(15):2171-2193

Krysl P (2007) Dynamically equivalent implicit algorithms for the integration of rigid body rotations. Int J Numer Methods Eng (in press). doi: $10.1002 / \mathrm{cnm} .963$

Kuipers JB (1999) Quaternions and rotation sequences. Princeton University Press, Princeton

LeeT, Leok M, Harris McClamrock N (2007) Lie group variational integrators for the full body problem. Comput Methods Appl Mech Eng 196:2907-2924

Lew A, Marsden JE, Ortiz M, West M (2003) Asynchronous variational integrators. Arch Rational Mech Anal 167:85-146

Lew A, Marsden JE, Ortiz M, West M (2004) Variational time integrators. Int J Numer Methods Eng 60:153-212

Lewis D, Simo JC (1996a) Conserving algorithms for the $\mathrm{N}$-dimensional rigid body. In: Integration algorithms and classical mechanics (Toronto, ON, 1993). Amer. Math. Soc., Providence, pp 121-139

Lewis D, Simo JC (1996b) Conserving algorithms for the $\mathrm{N}$-dimensional rigid body. Fields Inst commun 10:121-139

Maeda S (1980) Canonical structure and symmetries for discrete dynamics. Math Jpn 25:405-420

Marsden JE, Ratiu TS (1994) Introduction to mechanics and Symmetry, 1st edn. Springer, New York

Marsden JE, West $M$ (2001) Discrete mechanics and variational integrators. Acta Numer 10:357-514

Marsden JE, Pekarsky S, Shkoller S (1999) Discrete EulerPoincaré and Lie-Poisson equations. Nonlinearity 12:1647-1662

Moser J, Veselov AP (1991) Discrete versions of some classical integrable systems and factorization of matrix polynomilas. Commun Math Phys 139(2):217-243

Sanz-Serna JM, Calvo MP (1994) Numerical Hamiltonian problems. Chapman and Hall, London

Simo JC, Wong K (1991) Unconditionally stable algorithms for rigid body dynamics that exactly preserve energy and momentum. Int J Numer Methods Eng 31:19-52

Simo JC, Fox DD, Rifai MS (1990) On a stress resultant geometrically exact shell model. III. Computational aspects of the nonlinear theory. Comput Methods Appl Mech Eng 79(1):21-70

Simo JC, Tarnow N, Wong KK (1992) Exact energy-momentum conserving algorithms and symplectic schemes for nonlinear dynamics. Comput Methods Appl Mech Eng 100(1):63-116 Veselov AP (1988) Integrable discrete-time systems and difference operators. Funkts Anal Prilozhen 22(2):1-13

Wendlandt JM, Marsden JE (1997) Mechanical integrators derived from a discrete variational principle. Phys D 106(3-4):223-246 